\title{
Educação remota na continuidade da formação médica em tempos de pandemia: viabilidade e percepções
}

\author{
Distance Learning in continued medical training during the pandemic: feasibility and perceptions \\ Pedro Henrique dos Santos Silva' (D) ph_beta@ufpi.edu.br \\ Luciana Rocha Faustino' (1) Irfaustino@gmail.com \\ Maurício Santana de Oliveira Sobrinho ${ }^{2}$ (D) jornalistamauriciosantana@gmail.com \\ Franciele Basso Fernandes Silva' ${ }^{10}$ francibasso2@hotmail.com
}

\begin{abstract}
RESUMO
Introdução: Com a interrupção das atividades educacionais das universidades por causa da pandemia da Covid-19, formas de ensino virtuais, como a educação remota (ER), passaram a ser debatidas no meio acadêmico. No entanto, faz-se necessária uma avaliação criteriosa da ER antes de sua aplicação visando à qualidade do ensino.

Objetivo: Assim, o objetivo deste estudo foi avaliar a viabilidade da implantação da ER para discentes de um curso de Medicina.

Método: Aplicou-se um formulário on-line no qual constavam questões sobre aspectos demográficos e socioeconômicos, sobre acesso às tecnologias digitais e uma pergunta subjetiva relacionada ao uso da ER. Os dados quantitativos foram comparados pelos testes Kruskal-Wallis e Mann-Whitney com $\mathrm{p}<0,05$. Analisaram-se os dados qualitativos por meio do método de Classificação Hierárquica Descendente, e houve ainda uma análise pós-fatorial.

Resultados: O perfil socioeconômico de 266 participantes foi variado, sendo a maioria dos discentes brasileiros do sexo masculino, adultos jovens, cor da pele parda e renda familiar média elevada. Todos relataram ter acesso à energia elétrica, mas não à água encanada, à coleta pública de lixo e ao esgotamento sanitário. Todos possuíam pelo menos um equipamento eletrônico para acesso à internet, mas com variação no tipo e na velocidade da conexão. Um total de $80,8 \%$ dos discentes avaliou como viável a implantação da ER para o seguimento do curso. No entanto, 8,65\% dos discentes afirmaram que os equipamentos e a internet disponíveis não permitiriam o acompanhamento das atividades on-line. Foi observada uma correlação significativa entre a velocidade de acesso à internet e a renda familiar média, a cor da pele e o local da residência $(p<0,05)$, bem como entre possuir equipamentos adequados à ER e a renda familiar média $(p<0,05)$. A análise da pergunta objetiva revelou seis categorias: a necessidade de organização dos procedimentos e o anseio pela volta à normalidade; custos e benefícios da ER; planejamento e garantia da acessibilidade de todos os estudantes à internet de qualidade; acreditar que a realização da ER não é a solução para o problema; capacitação de professores e acadêmicos para o uso das plataformas digitais; e o querer realizar a ER; surgindo uma variedade de opiniões, visões e realidades pelos discentes.
\end{abstract}

Conclusão: Apenas o seguimento da ER, sem garantia de acesso para todos os discentes, é insuficiente e necessita da intervenção dos gestores para não haver prejuízo na aprendizagem daqueles menos favorecidos.

Palavras-chave: Educação; Educação de Graduação em Medicina; Saúde Pública; Infecções por Coronavírus; Educação a Distância; Tecnologia.

\section{ABSTRACT}

Introduction: With the interruption of University teaching activities due to the COVID-19 pandemic, various virtual learning strategies, such as Distance Learning (DL), have be discussed in the academic environment. However, before applying such strategies, a careful evaluation is required in order to ensure high quality learning.

Objective: The aim of this study was to evaluate the feasibility of implementing DL for students at a medical school.

Method: An online form was applied containing a demographic questionnaire, socioeconomic questionnaire, a questionnaire on access to digital technologies and a subjective question related to the use of DL. Quantitative data were compared using the Kruskal-Wallis or Mann-Whitney test, with $p<0.05$. Qualitative data were analyzed using the Descending Hierarchical Classification method and post-factorial analysis.

Results: The socioeconomic profile of 266 participants was varied, with most of the students being young adults, Brazilian and male with brown skin color and a high average family income. All students reported having access to electricity, but not running water, public garbage collection and sanitation. All had at least one electronic equipment for internet access, but with variations in the type and speed of internet connection. 80.8\% of the students considered the implementation of DL feasible for following the course. However, $8.65 \%$ stated that the equipment and the available internet equipment would not allow them to follow online activities. A significant correlation was observed between internet access speed and the average family income, skin color and the place of residence $(p<0.05)$; as well as between having equipment suitable for $D L$ and the average family income $(p<0.05)$. Analysis of the objective question revealed six categories: the need to organize procedures and the desire to return to normality; DL costs and benefits; planning and ensuring the accessibility of all students to quality internet; to believe that the realization of DL is not the solution to the problem; training teachers and academics to use digital platforms; and wanting to perform the DL; giving rise to a variety of opinions, views and realities from the students. Conclusion: Merely carrying on with DL, without any guaranteed access for all students, is insufficient and requires the intervention of managers to avoid detrimental consequences for the learning of those less favored.

Keywords: Education; Undergraduate Medical Education; Public Health; Coronavirus Infections; Distance Learning; Technology.

\footnotetext{
${ }^{1}$ Universidade Federal do Delta do Parnaíba, Parnaíba, Piauí, Brasil.

2 Universidade Federal do Piauí, Teresina, Piauí, Brasil.

Editora-chefe: Rosiane Viana Zuza Diniz ～～～Editor associado: Kristopherson Lustosa Augusto
}

Recebido em 29/09/20; Aceito em 18/01/21. | Avaliado pelo processo de doube blind review. 


\section{INTRODUÇÃO}

A pandemia da coronavirus disease 2019 (Covid-19), que rapidamente se tornou uma emergência global pelo alto potencial de transmissibilidade do severe acute respiratory syndrome coronavirus (Sars-CoV-2) 1-3, levou governos e instituições, em todo o mundo, a adotar medidas de controle que modificaram acentuadamente o modo de vida das pessoas ${ }^{4-6}$. Com o objetivo de evitar locais de disseminação do novo vírus e assim conter o avanço da pandemia, governos e organizações limitaram o funcionamento de diversos estabelecimentos e serviços ${ }^{4,7}$.

Ossistemaseducacionaisforam um dos serviçosquetiveram seu funcionamento interrompido por tempo indeterminado. Dessa forma, universidades, faculdades, institutos e escolas, públicos e privados, tiveram que paralisar suas atividades ${ }^{8,9} \mathrm{em}$ meados de março de 2020, o que afetou $90 \%$ dos estudantes em todo o mundo, segundo a Organização das Nações Unidas para a Educação, a Ciência e a Cultura (United Nations Educational, Scientific and Cultural Organization -Unesco) $)^{10}$.

No meio acadêmico, iniciaram-se debates sobre a implementação de alternativas que permitissem o seguimento das atividades de educação, especialmente aquelas relacionadas à formação de profissionais da saúde, sem que houvesse prejuízo às medidas de isolamento social instaladas e à qualidade do ensino-aprendizagem. Entre as possíveis alternativas, destacam-se as aulas em plataformas digitais como a educação remota $(E R)^{8}$. A ER se baseia no desenvolvimento de atividades educacionais sem a necessidade de presença física ou aglomeração de pessoas. Esse modelo de educação utiliza tecnologias digitais para promover a transmissão de informações entre discentes e docentes em tempo real ${ }^{11}$. Para o pleno desenvolvimento da ER, todos os participantes precisam ter acesso a equipamentos eletrônicos, como smartphones, computadores, notebooks ou tablets, bem como acesso à internet com velocidade adequada ${ }^{12,13}$.

Assim, diante da grande heterogeneidade socioeconômica da população universitária, torna-se necessária uma avaliação prévia, criteriosa e abrangente do acesso dos discentes aos meios necessários para implementação da ER. Nesse intuito, o objetivo do presente estudo foi analisar, na percepção dos acadêmicos do curso de bacharelado em Medicina de uma universidade federal, a viabilidade da ER na formação médica e as propostas de solução à problemática gerada pela pandemia do novo coronavírus.

\section{MÉTODOS}

\section{Desenho do estudo, população e aspectos éticos}

Trata-se de um estudo descritivo, exploratório, observacional, transversal quanti-qualitativo desenvolvido com estratégia websurvey. Este estudo foi aprovado pelo Comité de Ética e Pesquisa da Universidade Federal do Piauí (UFPI) campus Ministro Petrônio Portela, Parecer n 4.204.264.

Como critérios de inclusão para compor a amostra do estudo, consideraram-se todos os 266 discentes regularmente matriculados no bacharelado em Medicina da Universidade Federal do Delta do Parnaíba (UFDPar), no Piauí, que cursavam entre o primeiro e o oitavo período. Excluíram-se da análise todos os alunos com pendências matriculares ou que estavam cursando os períodos que compõem o estágio curricular obrigatório de caráter essencialmente prático (entre o nono e o décimo segundo período).

\section{Coleta de dados e aplicação do questionário}

A plataforma escolhida para websurvey foi a confecção de um questionário utilizando a plataforma de gerenciamento de pesquisas Google Forms. O questionário foi enviado ao e-mail dos discentes, registrados no Sistema Integrado de Gestão de Atividades Acadêmicas (Sigaa) da instituição de ensino superior (IES), que atendiam aos critérios de inclusão da pesquisa. Após dez dias, prazo estipulado para a adesão dos discentes à pesquisa, realizou-se nova tentativa de contato com aqueles que não enviaram as respostas do questionário. Como forma de buscar esses alunos, utilizaram-se redes sociais, como WhatsApp e Instagram, para contato. Por fim, para buscar os alunos que ainda assim não responderam à pesquisa, foi feito contato por telefonema.

Previamente ao acesso às questões do formulário, disponibilizou-se o Termo de Consentimento Livre e Esclarecido (TCLE) para que os participantes tomassem ciência de todos os procedimentos que seriam realizados. O formulário de pesquisa foi estruturado em quatro etapas: questionário demográfico, questionário socioeconômico, questionário de acesso às tecnologias digitais e uma pergunta subjetiva relacionada ao uso da ER.

No questionário demográfico, consideraram-se os seguintes aspectos: período cursado pelo aluno, raça/cor de pele, idade, estado e cidade de origem, e sexo biológico. No questionário socioeconômico, foi perguntado sobre a quantidade de pessoas dividindo residência; a quantidade de pessoas dividindo a renda familiar; a propriedade da casa; se havia local adequado para a prática de atividades educacionais; a zona em que a residência se localiza; o acesso a saneamento básico; o nível de escolaridade dos pais ou responsáveis; se o discente possuía alguma atividade laboral e qual tempo destinado ao trabalho; e a renda familiar per capita. No questionário de acesso às tecnologias digitais, foi questionado como era o acesso à internet (tipo da internet, velocidade da rede e compartilhamento com outras pessoas); sobre a quantidade e os tipos de equipamentos possuídos; em 
quais dispositivos havia acesso à internet; a exclusividade no uso dos equipamentos; a possibilidade de realizar atividades educacionais remotas com os equipamentos que os discentes possuíam, na visão deles, bem como a opinião do acadêmico sobre a realização de atividades educacionais remotas.

A última etapa do questionário foi pensada como um espaço aberto para que os discentes deixassem comentários e opiniões sobre a ER e/ou qualquer outra possível solução, sem obrigatoriedade de resposta. O enunciado da questão foi o seguinte: "Dê sua opinião sobre uma possível solução ao problema enfrentado em poucas palavras. Essa resposta não é obrigatória".

\section{Análise dos dados}

Todos os dados coletados foram exportados para uma planilha do programa Microsoft Excel 2016 e passaram por conferência para verificação de inconsistências ou erros de preenchimento. A geração e o armazenamento das respostas subjetivas foram totalmente anônimos, e não houve qualquer tipo de modificação, influência ou interferência por parte dos pesquisadores.

Para a análise quantitativa, realizou-se inicialmente a estatística descritiva dos dados. Logo após, os dados foram avaliados quanto à normalidade pelo teste estatístico de Kolmogorov-Sminorv com correção de significância de Lilliefors. As associações entre as variáveis foram avaliadas pelos testes Kruskal-Wallis e Mann-Whitney, e, quando necessário, realizou-se a interpretação das associações estatisticamente significativas com auxílio de tabelas cruzadas. As análises foram realizadas no software IBM SPSS (versão 22, IBM), estabelecendo um nível de significância de 5\%.

Para a análise qualitativa, os dados obtidos no estudo foram analisados por meio do software IRaMuTeQ, ancorado no ambiente estatístico do software $\mathrm{R}$ e na linguagem Python, e transformados em corpus textual. Após transformação dos dados, utilizou-se o método de Classificação Hierárquica Descendente (CHD), no qual os segmentos textuais são classificados em função dos seus respectivos vocabulários, e o conjunto de segmentos foi repartido com base na frequência de formas reduzidas para obter classes de Unidades de Contexto Elementares (UCE), o que permite identificar a quantidade de palavras presentes nos discursos e a frequência média de sua ocorrência. Ao mesmo tempo, apresentaram-se os vocabulários semelhantes entre si e aqueles diferentes das UCE das outras classes ${ }^{14}$.

Realizou-se ainda a análise fatorial de correspondência feita a partir da CHD (análise pós-fatorial), com a distribuição, num plano cartesiano, das diferentes palavras e variáveis associadas a cada uma das classes obtidas. Essa análise possibilitou a recuperação dos segmentos de texto associados a cada classe, levando à identificação do contexto das palavras estatisticamente significativas, o que favoreceu a categorização e a classificação das respostas dos discentes por meio de análise qualitativa.

Após a etapa de construção da CHD, realizou-se a exploração do corpus textual buscando um recorte temático das falas dos acadêmicos, isto é, uma divisão das falas de acordo com a ideia que elas expressam:"concordar com a ER", "discordar da ER", por exemplo. Após a análise, foi realizada associação entre a resposta dada à pergunta subjetiva e as repostas dadas às seguintes perguntas do questionário objetivo: "Você acredita que os equipamentos que possui lhe permitiriam realizar as atividades acadêmicas da ER?" e "Você acredita que a implantação da ER é uma boa solução no momento atual?". Após essa associação, criaram-se quatro grupos de respostas: 1. "Não possui equipamentos suficientes, mas acredita que a ER é uma boa alternativa"; 2. "Não possui equipamentos suficientes e não acredita que a ER é uma boa alternativa"; 3 . "Possui equipamentos suficientes, mas não acredita que a ER é uma boa alternativa”; e 4. "Possui equipamentos suficientes e acredita que a ER é uma boa alternativa".

Para garantir o completo sigilo e anonimato, as falas dos participantes foram identificadas pela letra $D$ de discente, seguida de um número que correspondeu à sequência de inclusão no estudo (por exemplo D1, D2, ... e D15).

\section{RESULTADOS}

\section{Dados socioeconômicos e demográficos e acesso às tecnologias digitais}

Dos 266 participantes considerados elegíveis, 163 $(61,27 \%)$ foram do sexo masculino e 103 (38,73\%) do sexo feminino. A média de idade observada foi de 23,39 anos, sendo $14,66 \%$ adolescentes ( $<20$ anos), 66,54\% adultos jovens (entre 20 e 25 anos) e 18,8\% com mais de 25 anos. Em relação à cor de pele, 144 (54,13\%) se autodeclaram pardos, 94 (35,33\%) brancos, $24(9,2 \%)$ pretos e quatro $(1,5 \%)$ preferiram não declarar a sua cor de pele.

A amostra avaliada incluiu discentes com origem em três países (Brasil, República de Cabo Verde e Guiné-Bissau), sendo a grande maioria composta por brasileiros de 22 estados federativos, dos quais 245 (92,1\%) de regiões urbanas, e, destes, 123 eram provenientes de capitais. Foi observado que a maioria dos discentes não trabalha (84,96\%). Entretanto, entre os que trabalham, a maioria continua exercendo suas atividades durante o período de pandemia. Entre os que permanecem trabalhando, 52\% têm cargas horárias de 20 horas ou mais por semana. Em relação à renda familiar média dos discentes, observou-se que a maioria (40,97\%) apresenta renda per capita elevada, superior a $\mathrm{R} \$ 1.500,00$ mensais. Contudo, uma parcela considerável (19,85\%) apresenta renda inferior a $\mathrm{R} \$ 600,00$ (Tabela 1). 
Tabela 1. Distribuição das características socioeconômicas e demográficas dos discentes do curso de bacharelado em Medicina da Universidade Federal do Delta do Parnaíba, Piauí, Brasil, 2020.

\begin{tabular}{lccc}
\hline & Indicadores & $\mathbf{n}(\mathbf{n}=\mathbf{2 6 6})$ & $\%$ \\
\hline Sexo & & & \\
& Masculino & 163 & 61,27 \\
& Feminino & 103 & 38,73 \\
\hline Idade & & & \\
& $<20$ anos & 39 & 14,66 \\
& 20 a 25 anos & 177 & 66,54 \\
& $>25$ anos & 50 & 18,80 \\
\hline Cor da pele & & & \\
& Parda & 144 & 54,13 \\
& Preta & 24 & 9,02 \\
& Branca & 94 & 35,33 \\
& Outra & 4 & 1,50 \\
\hline
\end{tabular}

Cidade de origem

\begin{tabular}{ccc} 
Capital & 123 & 46,24 \\
Interior & 143 & 53,76 \\
\hline
\end{tabular}

Região de origem

\begin{tabular}{ccc}
$\begin{array}{c}\text { Zona urbana } \\
\text { Zona rural }\end{array}$ & 245 & 92,10 \\
\hline Acesso a saneamento básico & 21 & 7,90 \\
Água encanada & 263 & \\
Energia elétrica & 266 & 100 \\
Esgotamento sanitário & 238 & 89,47 \\
Coleta pública de lixo & 253 & 95,11 \\
\hline
\end{tabular}

Propriedade da casa

\begin{tabular}{ccc} 
Própria & 182 & 68,42 \\
Cedida & 11 & 4,13 \\
Alugada & 73 & 27,44 \\
\hline
\end{tabular}

Trabalho durante a pandemia

Não trabalha

$226 \quad 84,96$

Não está trabalhando

Home office

2

0,75

Trabalho presencial

20

7,50

18

6,70

Tempo de trabalho durante a pandemia

\begin{tabular}{ccc} 
20 horas semanais ou menos & 18 & 6,70 \\
20 a 40 horas semanais & 16 & 6,01 \\
Mais de 40 horas semanais & 4 & 1,50 \\
\hline Renda familiar per capita & & \\
$<$ R\$ 150,00 & 5 & 1,87 \\
De R\$ 150,00 a R\$ 299,99 & 10 & 3,70 \\
De R \$300,00 a R\$ 599,99 & 38 & 14,28 \\
De R\$ 600,00 a R\$ 999,99 & 36 & 13,53 \\
De R\$ 1.000,00 a R\$ 1.500,00 & 68 & 25,56 \\
Mais de R\$ 1.500,00 & 109 & 40,97
\end{tabular}

Fonte: Elaborada pelos autores.
Tabela 2. Acesso à internet e a aparelhos eletrônicos pelos discentes do curso de bacharelado em Medicina da Universidade Federal do Delta do Parnaíba, Piauí, Brasil, 2020.

\begin{tabular}{ccc}
\hline Variáveis & $\mathbf{n}(\mathbf{n = 2 6 6 )}$ & $\%$ \\
\hline Acesso à internet & & \\
Sim & 266 & 100 \\
Não & 0 & 0 \\
\hline
\end{tabular}

\begin{tabular}{ccc}
\hline Tipo da internet & & \\
Banda larga ilimitada & 204 & 76,70 \\
Banda larga limitada & 52 & 19,50 \\
Internet móvel limitada & 10 & 3,80 \\
\hline Velocidade da internet & & \\
Não sabe informar & 43 & 16,16 \\
Menos de 1 Mbps & 14 & 5,26 \\
De 1 Mbps a 15 Mbps & 93 & 34,96 \\
Mais de 15 Mbps & 116 & 43,60 \\
\hline Quantas pessoas dividem a rede de internet & \\
Nenhuma & 13 & 4,80 \\
Uma & 18 & 6,70 \\
Duas & 40 & 15 \\
Três & 69 & 26 \\
Quatro ou mais & 126 & 47,50 \\
\hline
\end{tabular}

Aparelhos eletrônicos disponíveis

\begin{tabular}{ccc} 
Smartphone & 265 & 99,60 \\
Tablet & 69 & 25,90 \\
Notebook & 258 & 97 \\
Computador de mesa & 70 & 26,30 \\
\hline Quantidade de equipamentos com acesso à internet* & \\
Um & 21 & 7,90 \\
Dois & 161 & 60,50 \\
Três & 65 & 24,40 \\
Quatro & 19 & 7,20 \\
\hline Quantidade de equipamentos de uso exclusivo* & \\
Um & 57 & 21,40 \\
Dois & 182 & 68,40 \\
Três & 25 & 9,40 \\
Quatro & 2 & 0,8 \\
\hline Sim & & \\
Não & 243 & 91,35 \\
\hline Os seus equipamentos permitiriam ER & 23 & 8,65 \\
\hline
\end{tabular}

Quantidade de equipamentos que possuem acesso à internet*

$\begin{array}{ccc}\text { Um } & 21 & 7,80 \\ \text { Dois } & 161 & 60,5 \\ \text { Três } & 65 & 24,4 \\ \text { Quatro } & 19 & 7,10\end{array}$

*Considerou-se o acesso à internet por meio de smartphones, tablets, notebooks e computadores de mesa; **ER = educação remota.

Fonte: Elaborada pelos autores. 
A Tabela 2 apresenta os dados referentes ao acesso e à qualidade da internet, bem como ao acesso aos aparelhos eletrônicos que permitem conexão com a internet. Todos os discentes que compuseram a amostra afirmaram que possuem acesso à internet. Entretanto, foram observadas variações importantes quanto ao tipo e à velocidade da conexão, de forma que uma parcela considerável dos discentes $(23,3 \%)$ não tem acessoà internetilimitadae 56,39\% têm internet debaixa ou média velocidade ou não souberam informar a velocidade da conexão. Constatou-se também uma alta taxa de compartilhamento de rede, visto que $47,5 \%$ dos acadêmicos compartilham sua internet com quatro pessoas ou mais. Verificou-se ainda que todos os acadêmicos possuíam pelo menos um equipamento eletrônico que permita acesso à internet.
Ao serem questionados diretamente sobre a possibilidade de implantação da ER, 8,65\% dos discentes afirmaram que os equipamentos e a internet disponíveis não permitiriam acompanhar as atividades. Um total de $80,8 \%$ avaliou que é viável a implantação da ER para o seguimento do curso, enquanto 19,2\% mencionaram que é inviável.

A Tabela 3 apresenta a correlação entre a velocidade de acesso à internet e o acesso a equipamentos digitais que permitem o acompanhamento de atividades de ER, com as características demográficas e socioeconômicas dos discentes. Foi evidenciada correlação estatisticamente significativa entre a velocidade de acesso à internet e três variáveis: renda familiar média, cor da pele e local da residência $(p<0,05)$; bem como entre possuir equipamentos adequados à ER e a renda familiar

Tabela 3. Associação entre velocidade do acesso à internet, equipamentos que permitiriam $\mathrm{R}^{*}$ e fatores demográficos de discentes do curso de bacharelado em Medicina da Universidade Federal do Delta do Parnaíba, Piauí, Brasil, 2020.

\begin{tabular}{|c|c|c|c|c|c|c|c|c|}
\hline \multirow{3}{*}{ Variáveis } & \multicolumn{5}{|c|}{ Velocidade da internet } & \multicolumn{3}{|c|}{ Os equipamentos permitiriam $\mathrm{ER}^{*}$ ? } \\
\hline & $\begin{array}{c}\text { Não sei } \\
\text { informar }\end{array}$ & $\begin{array}{c}\text { Menos de } \\
1 \mathrm{Mbps}\end{array}$ & $\begin{array}{c}\text { De } 1 \text { Mbps a } \\
15 \text { Mbps }\end{array}$ & $\begin{array}{c}\text { Mais de } 15 \\
\text { Mbps }\end{array}$ & & Sim & Não & \\
\hline & $\%$ & $\%$ & $\%$ & $\%$ & Valor de $p$ & $\%$ & $\%$ & Valor de $p$ \\
\hline \multicolumn{9}{|l|}{ Sexo } \\
\hline Masculino & 14,7 & 4,9 & 37,4 & 42,9 & \multirow{2}{*}{$p>0,05$} & 92,0 & 8,0 & \multirow{2}{*}{$p>0,05$} \\
\hline Feminino & 18,4 & 5,8 & 31,1 & 44,7 & & 90,3 & 9,7 & \\
\hline$<20$ anos & 20,5 & 0,0 & 53,8 & 25,6 & \multirow{3}{*}{$p>0,05$} & 94,9 & 5,1 & \multirow{3}{*}{$p>0,05$} \\
\hline Entre 20 e 25 anos & 16,4 & 6,8 & 31,1 & 45,8 & & 90,4 & 9,6 & \\
\hline$>25$ anos & 12,0 & 4,0 & 34,0 & 50,0 & & 92,0 & 8,0 & \\
\hline \multicolumn{9}{|l|}{ Cor da pele } \\
\hline Outro & 0,0 & 25,0 & 0,0 & 75,0 & $p<0,05$ & 100,0 & 0,0 & $p>0,05$ \\
\hline \multicolumn{9}{|l|}{ Cidade de origem } \\
\hline Capital & 16,3 & 3,3 & 30,1 & 50,4 & \multirow{2}{*}{$p>0,05$} & 91,9 & 8,1 & \multirow{2}{*}{$p>0,05$} \\
\hline Interior & 16,1 & 7,0 & 39,2 & 37,8 & & 90,9 & 9,1 & \\
\hline \multicolumn{9}{|l|}{ Local da casa } \\
\hline Zona urbana & 17,1 & 4,5 & 33,1 & 45,3 & \multirow{2}{*}{$\mathrm{p}<0,05$} & 91,0 & 9,0 & \multirow{2}{*}{$p>0,05$} \\
\hline Zona rural & 4,8 & 14,3 & 57,1 & 23,8 & & 95,2 & 4,8 & \\
\hline \multicolumn{9}{|l|}{ Renda familiar per capita } \\
\hline Menos de $\mathrm{R} \$ 150,00$ & 40,0 & 20,0 & 40,0 & 0,0 & $p<0,05$ & 80,0 & 20,0 & $p<0,05$ \\
\hline
\end{tabular}

*ER = educação remota.

Fonte: Elaborada pelos autores. 
média, especialmente demonstrando que indivíduos com renda familiar menor possuem menos acesso à internet de velocidade adequada e a equipamentos para a ER $(p<0,05)$. Quanto maior a renda dos indivíduos, melhor a velocidade de acesso à internet, com $56 \%$ dos discentes com renda familiar média acima de $R \$ 1.500,00$ possuindo internet com velocidade superior a $15 \mathrm{M}$ bps. Os discentes provenientes de zona rural, com cor de pele preta ou parda e com renda familiar média baixa apresentaram menores proporções de acesso à internet de alta velocidade.

\section{CHD: resultados da pergunta qualitativa}

Com a análise do corpus textual, produzido a partir das respostas dos discentes à pergunta subjetiva do formulário de pesquisa, obteve-se um total de quatro textos, com 79 segmentos de textos (ST) e aproveitamento de 50 ST. Emergiram 2.856 ocorrências (palavras, formas ou vocabulários), sendo 831 palavras distintas e 538 palavras que aparecem somente uma vez.

O conteúdo análisado foi dividio pelo software em cinco classes: 1. com dez ST (20\%); 2. com nove ST (18\%); 3. com 34 ST (28\%); 4. com 9 ST (18\%); e 5. com oito ST (16\%). Conforme observado na Figura 1, o grupo B foi mais significativo, totalizando $66 \%$ dos ST. A partir desse esquema hierárquico de vocabulários, foi possível inferir o conteúdo do corpus, nomear a classe e compreender os grupos de discurso, expressões e ideias.
A Figura 2 mostra o plano fatorial do corpus textual obtido, com a distribuição, num plano cartesiano, das diferentes palavras e variáveis associadas a cada uma das classes da CHD, apresentadas com o mesmo padrão de cores. A distribuição espacial permite a avaliação da relação entre as palavras das classes pela proximidade e pela frequência de ocorrência apontada pelo tamanho das palavras.

No eixo $X$, que representa $32,52 \%$ de distribuição do corpus textual, a classe 1 (eixo $\mathrm{X}$ negativo) é claramente separada das classes 2 e 5 (eixo $X$ positivo). Em adição, há maior predomínio de posicionamento central das classes 3 e 4 nesse mesmo eixo. No eixo $Y$, que representa $24,85 \%$ de distribuição no corpus textual, as classes 3 (eixo Y negativo), 5 (eixo Y positivo) e 4 (eixo Y positivo) apresentam-se claramente separadas.

A análise crítica dos pesquisadores sobre o conteúdo produzido permitiu o agrupamento das respostas dos discentes, revelando seis categorias. Cada categoria foi construída pela associação da análise das respostas dos discentes em conjunto com a frequência de palavras listadas de acordo com a CHD e pela apresentação do plano fatorial.

\section{A necessidade de organização dos procedimentos e o anseio pela volta à normalidade}

A grande maioria dos discursos apresentados pelos acadêmicos, sejam eles favoráveis ou não à implementação da

Figura 1. Dendrograma da CHD do corpus textual de discentes de medicina da Universidade Federal do Delta do Parnaíba, Piauí, Brasil, 2020.

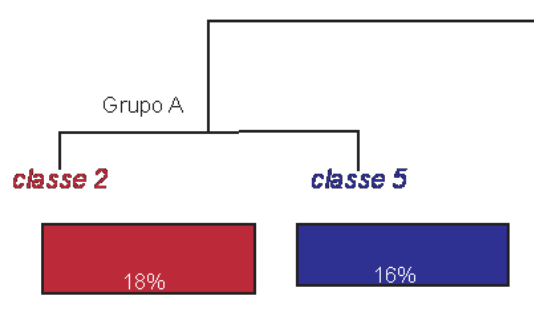

$\begin{array}{ll}\text { universidad } & \text { gravar } \\ \text { internet } & \text { acesso } \\ \text { aluno } & \text { aula } \\ \text { plano } & \text { realizar } \\ \text { exemplo } & \text { forma } \\ \text { acesso } & \text { plataforma } \\ \text { possuir } & \text { online } \\ \text { utilizar } & \text { período } \\ \text { condição } & \text { atividade } \\ \text { atividade } & \text { ficar } \\ \text { dever } & \text { utilizar } \\ \text { ufdpar } & \text { acontecer } \\ \text { acreditar } & \\ \text { necessitar } & \\ \text { ajudar } & \\ \text { acontecer } & \\ \text { acompanhar } & \end{array}$



medicina
curso
ufdpar
achar
caso
atividade
medida
ajudar
cursar

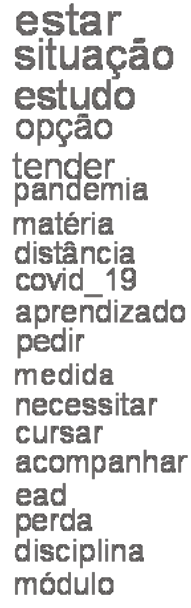

estar solução realizaçāo semestre ead achar módulo ciclo acreditar momento realizar estudo opcảo tender matéria distância aprendizado medida necessitar cursar ead disciplin módulo

Fonte: Software IRaMuTeQ. 
Figura 2. Plano fatorial do corpus textual de discentes de medicina da Universidade Federal do Delta do Parnaíba, Piauí, Brasil, 2020.



Fonte: Software IRaMuTeQ.

ER como forma de seguimento das atividades educacionais, mostrou uma significativa preocupação quanto à necessidade de uma análise situacional ampla, especialmente no que concerne ao acesso de todos a condições que permitam acompanhar as atividades desenvolvidas.

[...] deve ser traçado um planejamento acerca da situação que vivemos e as particularidades de alguns alunos. Podem ser contratados serviços específicos para as pessoas do curso que não têm acesso à internet (acredito ser minoria, pelos conhecimentos que tenho) e medidas extraordinárias tomadas. Algumas matérias podem sim ser feitas à distância até a situação se normalizar. Seria interessante que o período não seja perdido, mas reaproveitado [...] (D1).

[...] só deve ser implantado a ER caso todos os estudantes tenham acesso a tecnologia suficiente para aproveitá-la [...] (D2).

\section{Custos e benefícios da ER}

O receio de prejuízos e atrasos na formação acadêmica também foi um ponto muito abordado nos textos apresentados. Para embasar suas falas, os acadêmicos buscaram também descrever experiências bem-sucedidas vistas em outras instituições, recomendando modelos de ação para a universidade.

[...] seria muito bom as aulas remotas pra diminuir as perdas educacionais. Porém, tiraria o direito as aulas a quem não tem acesso à internet e equipamentos. Para isso, acredito que a UFDPar poderia seguir o exemplo de universidades como a UFC que mapeou esses alunos e contratou planos de internet e ofereceu equipamentos tecnológicos [...] (D3).

\section{Planejamento e garantia da acessibilidade de todos os estudantes à internet de qualidade}

A sugestão de uso da internet como uma alternativa também é tema bastante apontado pelos acadêmicos, sendo relevante a visão deles ao cogitarem essa possibilidade como real e que deve ser debatida.

[...] como sugestão aos alunos que porventura possam estar impossibilitados de acesso à internet, pode-se utilizar o exemplo de outras UFs que JÁ estão promovendo planos assistenciais a essa parcela do corpo discente, com distribuição de pacotes de dados de internet adquiridos pela Universidade para os alunos que realmente necessitem [...] (D4). 
[...] várias aulas, paralelas estão sendo realizadas por vídeo, como aulas em liga ou congressos e outras faculdades. A Universidade devia pegar bons exemplos positivos para usar como parâmetro [...] (D5).

\section{Acreditar que a realização da ER não é a solução para o problema}

Não fazer ER. Aguardar as recomendações para voltar presencialmente quando possível e seguro [...] (D6).

Entre as opiniões expressas, diversos alunos se manifestaram de forma contrária à implantação da ER. Dentre os diversos motivos apontados, o mais frequente foi a falta de garantia do acesso de todos às atividades desenvolvidas, especialmente pela ampla variedade socioeconômica observada entre os alunos do curso, mas também por questões pessoais.

[...] a implementação da ER é injusta com a parcela de alunos que não poderia acompanhar as atividades por falta de internet ou aparelhos [...] (D7).

Eu não tenho internet suficiente para realização de atividades online (D8).

Nem todos alunos possuem internet de qualidade para a ER e a realização de práticas ficaria prejudicada [...] (D9).

\section{Capacitação de professores e acadêmicos para o uso das plataformas digitais}

A necessidade de capacitação de professores e discentes em relação às plataformas que são utilizadas para o desenvolvimento da ER e o envolvimento deles em atividades extra-acadêmicas condicionadas pela pandemia foram importantes pontos levantados para contrapor a proposta.

O preparo dos alunos e dos professores para a aplicação de ensino remoto não pode ser feita de uma hora pra outra. Já vivenciamos diversos problemas com aulas presenciais, imagine em ER... A meu ver, essa não é uma solução viável [...] (D11).

Nossos professores estão trabalhando contra a covid-19, os casos estão aumentando, tem gente que está em casa tendo que ajudar os pais trabalhando porque a situação não está fácil. A pandemia vai nos fazer perder aulas sim, infelizmente. Ninguém pediu por isso (D10).

Além disso, outro fator apontado foi a necessidade de se levar em consideração os aspectos psicossociais dos envolvidos nesse processo.

Eu tenho condições pra fazer, mas tenho colegas que não têm. Psicologicamente falando, também é complicadíssimo pra quem está sofrendo [...] (D10).
[...] um momento de emoções e angústias enfrentadas por todos durante esse período difícil [...] (D4).

\section{O querer realizar a ER}

Muitos alunos também tiveram opiniões de apoio à implantação da ER como forma de promover o seguimento das atividades acadêmicas. Entre as diversas justificativas para as opiniões apresentadas, destacaram-se as seguintes: o medo de que haja atrasos na formação dos discentes, a visão de que a ER é a única solução para o seguimento das atividades acadêmicas e a percepção de que não há outra solução que permita manter as medidas de distanciamento social.

Sou plenamente favorável à implementação das aulas em ER com a máxima urgência possível, evitando assim a perda do semestre que já havia iniciado [...] em vista do período difícil que estamos vivendo seria muito bom as aulas ER pra diminuir as perdas educacionais [...] (D12).

AER é a solução inevitável, visto que o fim da pandemia ainda é indeterminado [...] (D13).

[...] caso a quarentena ainda dure muito, infelizmente é o melhor a ser feito [...] (D14).

Além disso, em meio às opiniões, os alunos utilizaram o espaço para dar ideias de como lidar com a situação e permitir que as dificuldades apresentadas pela ER sejam mitigadas.

\begin{abstract}
Uma possível solução é o oferecimento de pacotes de internet para as pessoas que dispõem unicamente de redes móveis para acesso à internet com o objetivo de facilitar o acesso. Outra alternativa é a gravação das aulas online em vídeos de forma a facilitar o acesso posterior, em caso de perda de conexão e que, somado a isso, houvesse um controle das pessoas que puderam assistir, mesmo que em outro horário [...] (D15).

[...] outros planos, como auxílio financeiro por meio de bolsas estudantis, podem ser tentados, para possibilitar o acesso à internet e a um computador por parte do aluno [...] (D4).
\end{abstract}

\section{DISCUSSÃO}

Os resultados apresentados demonstram um perfil socioeconômico variado, bem como uma ampla gama de opiniões, visões e realidades vivenciadas pelos discentes ante a problemática gerada pela pandemia da Covid-19.

Com relação ao perfil socioeconômico, observou-se que a maior parte dos acadêmicos que compuseram a amostra possui renda familiar média elevada. Apesar do processo de transformação do perfil socioeconômico das populações universitárias visto nas últimas duas décadas, especialmente como consequência do advento das ações afirmativas que buscam a promoção de condições equânimes de acesso à 
universidade para todos os grupos sociais e raciais, aindaé possível perceber que discentes de baixa renda são minoria nos cursos de Medicina ${ }^{15,16}$. O cenário descrito no presente estudo corrobora os achados presentes na literatura nacional. Veras et al. ${ }^{17}$, em estudo que avaliou o perfil socioeconômico de acadêmicos de Medicina em uma universidade federal do Nordeste brasileiro, observaram que mais da metade da população era composta por indivíduos com renda familiar elevada. A contradição do efeito das cotas sociais sobre o acesso aos cursos de Medicina por universitários de renda menos elevada se torna mais evidente quando se observa que $69,7 \%$ dos graduandos brasileiros possuem renda familiar mensal inferior a cinco salários mínimos ${ }^{18}$. Ristoff ${ }^{19}$ descreve que os cursos de Medicina no Brasil são aqueles em que há maior proporção de acadêmicos de alta renda, embora haja tendência de queda desse padrão. Apesar de o cenário descrito em nosso trabalho seguir a tendência dos estudos com cursos de Medicina ${ }^{17,20,21}$, os resultados apontam que uma parcela considerável dos discentes não possui condições socioeconômicas favoráveis, incluindo indivíduos com renda mensal per capita interior a $\mathrm{R} \$ 150,00$. Essa realidade corrobora o que foi observado em nosso estudo por meio dos discursos apresentados por alguns discentes, em que o conhecimento da realidade dos colegas de curso foi fator determinante para que alguns, mesmo possuindo condições de acompanhar atividades de ER, se posicionassem contra a aplicação desse recurso.

Observou-se em nosso estudo relação significativa entre a renda familiar dos discentes e a velocidade da internet à qual têm acesso, e ao acesso a equipamentos que permitiriam a ER. A heterogeneidade observada na distribuição dos recursos financeiros e das tecnologias digitais necessários ao desenvolvimento da ER expõe um cenário em que não há garantia de que todos poderão ter acesso de qualidade às atividades de educação que venham a ser desenvolvidas por meio de plataformas eletrônicas ${ }^{22}$. Isso ocorre porque, como descrevem Oliveira et al. ${ }^{23}$, a desigualdade socioeconômica se torna determinante para que intervenções educacionais impactem os diversos grupos sociais de formas distintas. No Brasil, país que tem raízes fincadas em uma história escravocrata, cujas consequências reverberam na sociedade até os tempos atuais, pessoas de pele negra são mais representadas por indicadores sociais negativos, como atividade no mercado de trabalho informal, o que limita o acesso a direitos básicos, como o acesso a bens, serviços e oportunidades ${ }^{24}$. Essa diferença no acesso a formas de ascensão social se manifesta em diversos âmbitos da sociedade, incluindo o meio acadêmico. Cursos acadêmicos vistos socialmente com maior prestígio tendem a ser majoritariamente ocupados por pessoas brancas ${ }^{16}$. Os resultados do presente estudo corroboram esse achado por demonstrarem, com relação estatisticamente significativa, que pessoas de pele parda e negra possuem menos acesso à internet de velocidade adequada que pessoas de pele branca e, consequentemente, menor possibilidade de desenvolvimento de atividades de ER.

Os resultados aqui apresentados demonstraram que todos os discentes que compuseram a amostra possuem acesso à internet. Esse achado é muito superior aos dados apresentados pelo Instituto Brasileiro de Geografia e Estatística (IBGE), obtidos por meio da Pesquisa Nacional por Amostra de Domicílios Contínua, que mostram que somente $79,1 \%$ dos domicílios brasileiros possuíam acesso à internet no ano de $2018^{25}$. Rego et al. ${ }^{21}$, em estudo com discentes de Medicina de uma universidade federal do Norte do país, observaram que $93,5 \%$ dos alunos tinham acesso à internet. Achados descritos por outros estudos nacionais e internacionais com populações universitárias, entretanto, apontam níveis mais elevados de acesso, o que corrobora os resultados do presente estudo e demonstra a heterogeneidade do acesso a essa tecnologia ${ }^{26-28}$. Apesar do constante desenvolvimento tecnológico vivenciado pela sociedade brasileira e do aumento significativo do acesso a tecnologias digitais por parte da parcela da população economicamente menos favorecida, o acesso à internet de qualidade e a utilização efetiva de suas potencialidades ainda não representam a realidade de todos os cidadãos ${ }^{29}$. Em uma sociedade cada vez mais fundamentada em compartilhamento de saberes e informações, as tecnologias digitais são responsáveis por inserir e transformar aqueles que podem produzir e consumir conteúdos, mas também podem tornar ultrapassados e excluir aqueles sem essa possibilidade ${ }^{30}$.

Apesar do acesso à internet por todos os discentes, observou-se que menos da metade deles possui uma velocidade de internet minimamente adequada ao $E R$, já que para essa modalidade de educação a internet de velocidade elevada é fundamental para garantir que os discentes tenham acesso às atividades de $E R$, uma vez que transmissões ao vivo de imagem e áudio demandam altas taxas de transferências de dados $^{31}$, o que se revela um importante fator de exclusão.

O presente estudo apontou ainda que os indivíduos que moram em zonas rurais ou cidades interioranas têm menor proporção de acesso à internet de alta velocidade. Essas regiões, por questões de custo, infraestrutura e logística, frequentemente possuem menor oferta de serviços que promovem inclusão digital e acesso a tecnologias digitais, comprovando que a região geográfica de moradia é fator que pode limitar o acesso às tecnologias digitais ${ }^{32}$. Estudos que avaliaram a apropriação dessas tecnologias no meio rural brasileiro demonstram um crescimento ao longo dos anos, mas o acesso a redes de internet ainda se mostra em escala modesta, especialmente quando comparada a grandes centros urbanos ${ }^{33,34}$. 
A globalização tem transformado a forma como as pessoas se comunicam e se relacionam, com efeitos também sobre a produção e disseminação de conhecimentos, o que torna cada vez mais evidente a ruptura de comunicação entre as elites e o restante da população ${ }^{35}$. A evolução desses processos cria constantemente novas assimetrias sociais, com fontes de inclusão e exclusão que precisam ser analisadas e definidas dentro de cada contexto no qual estão sendo observadas, tornando populações rurais, pelos motivos descritos, menos atingidas pelos efeitos do desenvolvimento tecnológico, e consequentemente com menor possibilidade de acesso a atividades de $\mathrm{ER}^{36}$.

Conforme esperado, a maior parcela da amostra analisada era composta por indivíduos que não trabalham. Entretanto, a inclusão integral dos acadêmicos nas atividades de ER passa também pela inclusão daqueles que possuem atividades laborais. Conciliar a dupla jornada de trabalho e estudos é uma tarefa árdua que pode levarà queda no desempenho acadêmico, fator que está frequentemente relacionado ao abandono das atividades educacionais ${ }^{37}$. A maior exposição de universitários ao estresse psicológico durante o período da pandemia pela Covid-19 pode, portanto, ser potencializado nesse grupo de discentes. Em razão disso, é imprescindível e urgente que haja ações voltadas ao atendimento dessa população com o objetivo de avaliar, de forma minuciosa, as condições de que os alunos dispõem de seguir as atividades de ER ${ }^{38}$.

Curiosamente, o discurso desenvolvido pelos discentes apresentou opiniões e abordagens muito distintas. Ao exporem o anseio pela volta das atividades educacionais, tornou-se clara a necessidade de que haja organização e cautela, com destaque especial à análise situacional de todos os acadêmicos, de modo que ninguém seja excluído do processo. A observação dos relatos expõe muita heterogeneidade de opiniões quanto à possibilidade de seguimento das atividades acadêmicas, e, para embasar suas falas, diversos argumentos foram levantados pelos alunos. Percebe-se, entretanto, um consenso quanto à necessidade de discutir a situação e fornecer à comunidade acadêmica uma posição clara que possa também servir de exemplo a outros cursos e instituições de ensino.

Além dos aspectos socioeconômicos e de acesso a tecnologias, a avaliação das afecções psicológicas dos discentes também é procedimento fundamental no entendimento da viabilidade da ER e não pode ser deixada de lado neste momento de pandemia ${ }^{39,40}$. Wang et al. ${ }^{41}$, em estudo que avaliou a resposta psicológica imediata da população à pandemia causada pela Covid-19, evidenciaram que ser estudante é fator significativamente relacionado ao surgimento de maiores níveis de ansiedade, depressão e estresse. As mudanças na rotina e nos hábitos de vida, bem como nas relações familiares e sociais geradas pela pandemia, são fatores que podem impactar negativamente a saúde mental e o bem-estar psicológico desses indivíduos ${ }^{42}$.

Nesse cenário, as opiniões dos acadêmicos se desenvolveram e se ramificaram em possibilidades opostas, mas baseadas num objetivo mútuo: o direito ao seguimento da educação de qualidade. Os argumentos utilizados para defender suas posições basearam-se em suas experiências e sentimentos. A vontade de retornar às atividades acadêmicas e dar continuidade à formação não impediu que os alunos mais abastados manifestassem empatia com os companheiros de curso que não têm condição de acessar a ER.

\section{CONCLUSÕES}

A avaliação do cenário educacional na formação médica, portanto, demonstra que o seguimento das atividades educacionais por meio da metodologia de ER é insuficiente, pois é impossível alcançar todos os envolvidos sem uma intervenção de gestores da instituição de ensino ou dos governantes.

O contexto analisado expõe uma ampla diversidade de realidades vividas pelos discentes do curso de bacharelado em Medicina da UFDPar. A pandemia da Covid-19 trouxe consigo incertezas e problemas que suscitam a organização e cautela na tomada de decisões sobre o seguimento das atividades acadêmicas, especialmente pela necessidade de incluir todos os indivíduos no processo educacional. A avaliação dos contextos vivenciados pelos discentes é ferramenta fundamental no direcionamento das medidas de gestão educacional com o objetivo de permitir igualdade e equidade no acesso ao direito à educação.

Diante disso, sugere-se que as entidades responsáveis (universidade e governo federal) elaborem estratégias que forneçam aos acadêmicos menos favorecidos aportes financeiros, tecnológicos e logísticos para garantir isonomia no acesso às atividades acadêmicas. A disponibilização de verbas para a aquisição de pacotes de internet e equipamentos eletrônicos adequados à realização de atividades educacionais é passo que deve ser dado com urgência para garantir o seguimento da formação médica.

\section{CONTRIBUIÇÃO DOS AUTORES}

Pedro Henrique dos Santos Silva contribuiu, de forma substancial, para a concepção e o delineamento do estudo, e participou da aquisição, análise e interpretação dos dados do trabalho, da elaboração de versões preliminares do artigo, da revisão crítica de importante conteúdo intelectual e da aprovação final da versão a ser publicada. Luciana Rocha Faustino participou da aquisição, análise e interpretação dos dados do trabalho, da revisão crítica de importante conteúdo intelectual e da 
aprovação final da versão a ser publicada. Maurício Santana de Oliveira Sobrinho participou da análise e interpretação dos dados do trabalho, e da aprovação final da versão a ser publicada. Franciele Basso Fernandes Silva contribuiu, de forma substancial, para a concepção e o delineamento do estudo, e participou da aquisição, análise e interpretação dos dados do trabalho, da revisão crítica de importante conteúdo intelectual e da aprovação final da versão a ser publicada. Em suma, todos os autores foram responsáveis por todos os aspectos do trabalho, no sentido de garantir que as questões relacionadas à exatidão ou à integridade de qualquer parte deste estudo fossem devidamente investigadas e resolvidas.

\section{CONFLITO DE INTERESSES}

Os autores declaram não haver conflito de interesses neste estudo.

\section{FINANCIAMENTO}

Declaramos que não houve financiamento para a realização desta pesquisa.

\section{REFERÊNCIAS}

1. Ge H, Wang X, Yuan X, Xiao G, Wang C, Deng T, et al. The epidemiology and clinical information about Covid-19. Eur J Clin Microbiol Infect Dis. 2010;39(6):1011-39.

2. Rothan HA, Byrareddy SN. The epidemiology and pathogenesis of coronavirus disease (Covid-19) outbreak. J Autoimmun. 2020;109:102433.

3. Sohrabi C, Alsafi Z, O'Neill N, Khan M, Kerwan A, Al-Jabir A, et al. World Health Organization declares global emergency: a review of the 2019 novel coronavirus (Covid-19). Int J Surg. 2020;76:71-6.

4. Garcia LP, Duarte E. Intervenções não farmacológicas para o enfrentamento à epidemia da Covid-19 no Brasil. Epidemiol Serv Saúde. 2020;29(2):1-4.

5. Silva PHS, Cirilo SSV, Soares LS, Silva FBF. Déficit e ocupação de leitos de unidade de terapia intensiva adulto do Sistema Único de Saúde no estado do Piauí sob a ótica da Covid-19. Vigilância Sanitária em Debate: Sociedade, Ciência \& Tecnologia. 2020;8(3):61-9.

6. Velavan TP, Meyer CG. The Covid-19 epidemic. Trop Med Int Health. 2020;25(3):278-80

7. Aquino EML, Silveira $I H$, Pescarini $J M$, Aquino R, Souza-Filho JA, Rocha AS, et al. Medidas de distanciamento social no controle da pandemia de Covid-19: potenciais impactos e desafios no Brasil. Ciênc Saúde Colet. 2020;25(supl 1):2423-46.

8. Vercelli LCA. Aulas remotas em tempos de Covid-19: a percepção de discentes de um programa de mestrado profissional em educação. Revista @mbienteeducação. 2020;13(2)47-60.

9. Bezerra ACV, Silva CEM, Soares FRG, Silva JAM. Fatores associados ao comportamento da população durante o isolamento social na pandemia de Covid-19. Ciênc Saúde colet. 2020;25(supl 1):2411-21.

10. Organização das Nações Unidas para a Educação, a Ciência e a Cultura. A Comissão Futuros da Educação da Unesco apela ao planejamento antecipado contra o aumento das desigualdades após a Covid-19. Unesco; 2020 [acesso em 6 ago 2020]. Disponível em: https://pt.unesco.org/news/ comissao-futuros-da-educacao-da-unesco-apela-ao-planejamentoantecipado-o-aumento-das.

11. Alves L. Educação remota: entre a ilusão e a realidade. Interfaces Científicas - Educação. 2020;8(3):348-65.
12. Araújo MS. EaD em tela: docência, ensino e ferramentas digitais. Revista Brasileira de Linguística Aplicada. 2014;14(3):735-41.

13. Echalar ADLF, Peixoto J, Echalar ADLF, Peixoto J. Programa Um Computador por Aluno: 0 acesso às tecnologias digitais como estratégia para a redução das desigualdades sociais. Ensaio: Avaliação e Políticas Públicas em Educação. 2017;25(95):393-413.

14. Camargo BV, Justo AM. IRAMUTEQ: um software gratuito para análise de dados textuais. Temas Psicol. 2013;21(2):513-8.

15. Guarnieri FV, Melo-Silva LL, Guarnieri FV, Melo-Silva LL. Cotas universitárias no Brasil: análise de uma década de produção científica. Psicol Esc Educ. 2017;21(2):183-93.

16. Souza PGA, Pôrto ACCA, Souza A, Silva Júnior AG, Borges FT, Souza $P G A$, et al. Perfil socioeconômico e racial de estudantes de medicina em uma universidade pública do Rio de Janeiro. Rev Bras Educ Med. 2020;44(3):e090.

17. Veras RM, Fernandez CC, Feitosa CCM, Fernandes S, Veras RM, Fernandez CC, et al. Perfil socioeconômico e expectativa de carreira dos estudantes de Medicina da Universidade Federal da Bahia. Rev Bras Educ Med. 2020;44(2):e056.

18. Franco AMP, Cunha S. Perfil socioeconômico dos graduandos da IFES. Radar, Tecnol Prod Comér Exter (Brasília). 2017;(49):13-19.

19. Ristoff D. The new profile of the Brazilian campus: an analysis of the socioeconomic profile of undergraduate students. Avaliação: Revista da Avaliação da Educação Superior (Campinas). 2014;19(3):723-47.

20. Fiorotti KP, Rossoni RR, Miranda AE. Perfil do estudante de Medicina da Universidade Federal do Espírito Santo, 2007. Rev Bras Educ Med. 2010;34(3):355-62.

21. Rego RM, Marques NA, Monteiro PC, Oliveira CLB, Lins NAA, Caldas CAM $O$ perfil atual do estudante de Medicina e sua repercussão na vivência do curso. Para Res Med J. 2018;2(1-4):e05.

22. Martelli Júnior $H$, Machado RA, Swerts MSO, Martelli DRB, Caldeira AP, Martelli Júnior $\mathrm{H}$, et al. The works of Hercules and Covid-19 lessons for medical education. Rev Bras Educ Med. 2020;44(3):e084.

23. Oliveira JBA, Gomes M, Barcellos T. A Covid-19 e a volta às aulas: ouvindo as evidências. Ensaio: Avaliação e Políticas Públicas em Educação. 2020;28(108):555-78.

24. Goes EF, Ramos DO, Ferreira AJF, Goes EF, Ramos DO, Ferreira AJF Desigualdades raciais em saúde e a pandemia da Covid-19. Trab Educ Saúde. 2020;18(3):e00278110.

25. Instituto Brasileiro de Geografia e Estatística. Pesquisa Nacional por Amostra de Domicílios Contínua. Acesso à internet e à televisão e posse de telefone móvel celular para uso pessoal - 2018. Rio de Janeiro: IBGE; 2020 [acesso em 14 jul 2020]. Disponível em: https://biblioteca.ibge.gov. br/index.php/biblioteca-catalogo?view=detalhes\&id=2101705.

26. Ayatollahi A, Ayatollahi J, Ayatollahi F, Ayatollahi R, Shahcheraghi SH Computer and internet use among undergraduate medical students in Iran. Pak J Med Sci. 2014;30(5):1054-8.

27. de Ávila GB, dos Santos ÉN, Jansen K, Barros FC. Internet addiction in students from an educational institution in Southern Brazil: prevalence and associated factors. Trends Psychiatry Psychother. 2020;42(4):30210 [acesso em 15 set 2020]. Disponível em: http://www.scielo.br/scielo. php?script=sci_abstract\&pid=S2237-60892020005007204\&lng=en\&nrm =iso\&tlng=en.

28. Kawyannejad R, Mirzaei M, Valinejadi A, Hemmatpour B, Karimpour HA, AminiSaman J, et al. General health of students of medical sciences and its relation to sleep quality, cell phone overuse, social networks and internet addiction. Biopsychosoc Med. 2019;13(1):1-7.

29. Mattos FAM, Chagas GJN. Desafios para a inclusão digital no Brasil. Perspect Ciência Inf. 2008;13(1):67-94.

30. Pischetola M. Inclusão digital e educação: a nova cultura da sala de aula. Petrópolis: Vozes; 2019. 187 p.

31. Arruda EP. Educação remota emergencial: elementos para políticas públicas na educação brasileira em tempos de Covid-19. EmRede. 2020;7(1):257-75. 
32. Viero VC, Silveira ACM. Apropriação de tecnologias de informação e comunicação no meio rural brasileiro. Cadernos de Ciência \& Tecnologia. 2011;28(1):257-77.

33. Lübeck E. A exclusão digital e a apropriação da internet no contexto rural brasileiro [dissertação]. Santa Maria: Universidade Federal de Santa Maria; 2004.

34. Viero VC. Tecnologias de informação e comunicação no contexto rural brasileiro: o modelo de monitoramento agrícola do Sistema Irriga ${ }^{\oplus}$ [dissertação]. Santa Maria: Universidade Federal de Santa Maria; 2009.

35. Silva Júnior AF, Oliveira AC, Souza LM, Menezes LDD. Inclusão social e inclusão digital em tempos de globalização: um estudo em uma escola rural. Em Extensão. 2010;9(1):83-98.

36. Guimarães JMM. Educação, globalização e educação a distância. Revista Lusófona de Educação. 2007;(9):139-58.

37. Pires LM, Souza MM, Medeiros M, Pires LM, Souza MM, Medeiros M. Aspectos de proteção e vulnerabilidade social de adolescentes de escola pública integral. Rev Bras Enferm. 2020;73(supl 1):e20190211.

38. Maia BR, Dias PC. Ansiedade, depressão e estresse em estudantes universitários: o impacto da Covid-19. Estud Psicol (Campinas). 2020;37:e200067.
39. Brooks SK, Webster RK, Smith LE, Woodland L, Wessely S, Greenberg N, et al. The psychological impact of quarantine and how to reduce it: rapid review of the evidence. The Lancet. 2020;395(10227):912-920.

40. Xiao C. A novel approach of consultation on 2019 novel coronavirus (Covid-19) - related psychological and mental problems: structured letter therapy. Psychiatry Investig. 2020;17(2):175-6.

41. Wang C, Pan R, Wan X, Tan Y, Xu L, Ho CS, et al. Immediate psychological responses and associated factors during the initial stage of the 2019 coronavirus disease (Covid-19) epidemic among the general population in China. Int J Environ Res Public Health. 2020;17(5)1-25.

42. Schmidt B, Crepaldi MA, Bolze SDA, Neiva-Silva L, Demenech LM, Schmidt $B$, et al. Saúde mental e intervenções psicológicas diante da pandemia do novo coronavírus (Covid-19). Estud Psicol (Campinas). 2020;37:e200063 [acesso em 8 set 2020]. Disponível em: http://www.scielo.br/scielo. php?script=sci_abstract\&pid=S0103-166X2020000100501\&lng=en\&nrm $=$ iso\&tlng=pt. 